Received: 2018.01.31 Accepted: 2018.04.26 Published: 2018.07.07

\title{
A Suspected Case of Hydralazine-Induced Hepatotoxicity: A Case Report and Review of Literature
}

Authors' Contribution:

Study Design A

Data Collection B

Statistical Analysis C

Data Interpretation D

Manuscript Preparation E

Literature Search F

Funds Collection G
ABCDEFG 1 Munish Sharma

EF 2 Milkeesso Foge

ADEF 3 Daniel Mascarenhas
1 Department of Internal Medicine, Easton Hospital, Easton, PA, U.S.A.

2 Department of Internal Medicine, Loma Linda University Medical Center,

Loma Linda, CA, U.S.A.

3 Department of Cardiology, Easton Hospital, Drexel University College of Medicine, Easton, PA, U.S.A.
Corresponding Author: Conflict of interest:
Munish Sharma, e-mail: munishintmed@gmail.com, munishs1@hotmail.com

None declared

\begin{abstract}
Patient: Male, 74
Final Diagnosis: Hydralazine induced hepatotoxicity

Symptoms: Abdominal discomfort

Medication: Hydralazine

Clinical Procedure: -

Specialty: Cardiology

Objective: Rare disease

Background:

Case Report:

Conclusions:

Hydralazine is an effective antihypertensive agent but may rarely have devastating hepatotoxic effects that are extremely variable, thus making the diagnosis difficult.

We report the case of a 74-year-old male patient who had transaminitis after being started on hydralazine by his cardiologist for poorly controlled hypertension. He had extreme dizziness, nausea, and weakness, which all resolved after discontinuation of hydralazine, and liver function test results also dramatically improved.

It is imperative that clinicians be aware of the possible hepatotoxicity of hydralazine and its clinical features so that the medication can be promptly discontinued to help promote liver recovery. This case report will add to the current literature about such infrequent cases of hydralazine-induced hepatotoxicity.
\end{abstract}

MeSH Keywords:

Full-text PDF:
Alanine Transaminase • Antihypertensive Agents • Hydralazine

https://www.amjcaserep.com/abstract/index/idArt/909279 


\section{Background}

Hydralazine is a phthalazine derivative and a commonly used antihypertensive drug. It essentially acts by causing peripheral vasodilation and is effective in controlling higher blood pressure. Hydralazine is well known to cause lupus-like syndrome and blood dyscrasias but it is also infrequently implicated in several forms of acute liver injury.

\section{Case Report}

A 74-year-old male patient with no history of alcohol abuse and with a past medical history of congestive heart failure (CHF), paroxysmal atrial fibrillation, coronary artery disease, and sick sinus syndrome status after permanent pacemaker implantation presented to our hospital on 12/18/17 with complaints of severe nausea, mild right upper-quadrant abdominal discomfort, dizziness, and worsening pedal edema. He was started on hydralazine $50 \mathrm{mg} 3$ times a day 2 months prior to this admission, for uncontrolled hypertension. On admission to our hospital, his blood pressure was $118 / 78 \mathrm{mmHg}$, he was afebrile, 12/min respiratory rate, $72 \mathrm{bpm}$ regular heart rate, and oxygen saturation of $98 \%$ at room air. Cardiovascular examination revealed S1-S2 with a 2-6 ejection systolic murmur along the left sternal border. Extremities had pitting pedal edema bilaterally. The patient had elevated liver function test results (Table 1), which were thought to be related to receiving amiodarone $200 \mathrm{mg}$ daily and atorvastatin $40 \mathrm{mg}$ daily, so these medications were discontinued on admission. He was also on Clopidogrel $75 \mathrm{mg}$ daily, furosemide $80 \mathrm{mg}$ daily, spironolactone $25 \mathrm{mg}$ daily, and propafenone $325 \mathrm{mg}$ twice daily. He opted to not take long-term oral anticoagulants since he was worried about bleeding complications. Notably, these were not new medications for him and he had been taking them for years. His alkaline phosphatase was 444 units/liter, ALT 132 units/liter, AST 262 units/liter, and total bilirubin was $4.0 \mathrm{mg} / \mathrm{dl}$ on admission. His hepatitis $B$ and $C$ serology were non-reactive, and results of anti-neutrophil antibodies (ANA) testing, anti-mitochondrial antibody testing, and actin antibody (IgG) testing were all negative. An ultrasound of the abdomen showed mild hepatomegaly with mild nonspecific periportal edema. No evidence of fatty liver was found. Ultrasound also showed gallbladder wall thickening without evidence of cholelithiasis or biliary dilatation. Cholescintigraphy ruled out acute cholecystitis. Total bilirubin was still high at $3.3 \mathrm{mg} / \mathrm{dl}$, and workup was done as an outpatient because he had significant relief of his presenting symptoms after treatment with IV diuresis. He was discharged on 12/23/2017. However, on $12 / 28$, he again called his cardiologist with complaint of extreme weakness and was admitted to the hospital. His total bilirubin was 4.4 this admission, in spite of discontinuing amiodarone and statin. Repeat ultrasound again showed no evidence of intra-or extra-hepatic biliary ductal dilation. There was no indication for endoscopic retrograde cholangiopancreatography (ERCP). He also underwent endoscopic ultrasound, which revealed a cystic lesion in the head of the pancreas $1 \mathrm{~cm} \times 9 \mathrm{~mm}$ in size, with slightly dilated pancreatic

Table 1. Biochemical parameters showing hepatocellular recovery.

\begin{tabular}{|c|c|c|c|}
\hline Chemistry & $\begin{array}{l}\text { Aspartate transaminase (AST) } \\
\text { inits/liter }\end{array}$ & $\begin{array}{c}\text { Direct bilirubin } \\
\text { (milligram/deciliter) }\end{array}$ & $\begin{array}{c}\text { Total bilirubin } \\
\text { (milligram/deciliter) }\end{array}$ \\
\hline $1 / 26 / 2018$ & 62 & & 1.6 \\
\hline $1 / 23 / 2018$ & 71 & 1.0 & 1.7 \\
\hline $1 / 22 / 2018$ & 89 & & 2.3 \\
\hline $1 / 2 / 2018$ & 108 & 2.1 & 3.5 \\
\hline $1 / 1 / 2018$ & 115 & & 3.5 \\
\hline $12 / 31 / 2017$ & 123 & 2.1 & 3.7 \\
\hline $12 / 30 / 2017$ & 117 & & 3.8 \\
\hline $12 / 29 / 2017$ & 122 & & 3.6 \\
\hline $12 / 28 / 2017$ & 166 & 2.4 & 4.4 \\
\hline $12 / 23 / 2017$ & 194 & & 3.3 \\
\hline $12 / 22 / 2017$ & 163 & & 2.9 \\
\hline $12 / 21 / 2017$ & 199 & 1.8 & 3.2 \\
\hline $12 / 20 / 2017$ & 174 & & 3.1 \\
\hline $12 / 19 / 2017$ & 177 & 2.3 & 3.3 \\
\hline $12 / 18 / 2017$ & 262 & 2.7 & 4.0 \\
\hline
\end{tabular}




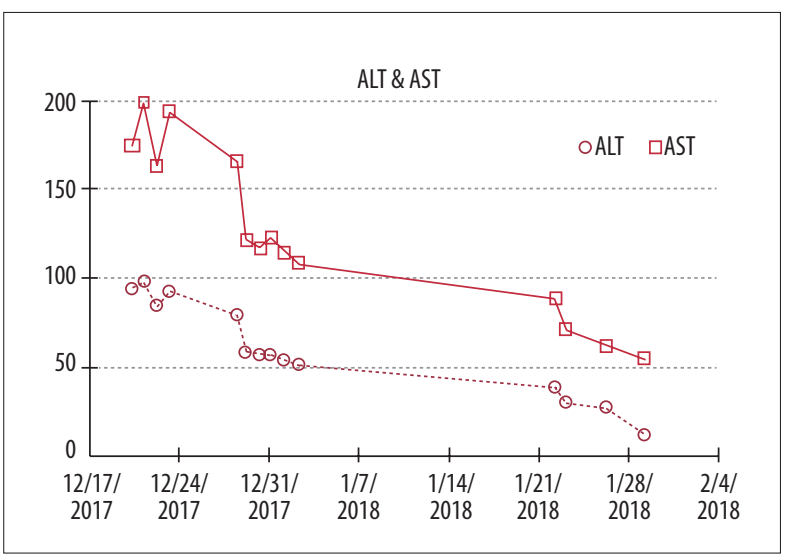

Figure 1. Trend of alanine transaminase (ALT, shown by dotted line) and aspartate transaminase (AST, shown by solid line).

duct possible side-branch intra-papillary mucinous neoplasm (IPMN). No evidence of a pancreatic head mass was seen. Again, there was no evidence of common bile duct obstruction. In view of possible cholestasis jaundice, hydralazine was discontinued on $12 / 28$. The patient was discharged from the hospital on 1/3/18 after his nausea, weakness, and dizziness resolved. His liver function tests were serially followed up as an outpatient and were found to have trended down gradually (Table 1; Figures 1, 2).

\section{Discussion}

Hydralazine belongs to the hydrazinophthalazine group of medications [1]. It is a direct-acting smooth-muscle relaxant and acts by decreasing peripheral vascular resistance, which leads to reduction in afterload as well blood pressure [2]. Hydralazine was first discovered by scientists at Ciba and was initially called C-5968 and 1-hydrazinophthalazine [3]. Due to its prominent reflex sympathetic stimulation of heart and resultant tachycardia, hydralazine is not used as a first-line antihypertensive agent [3]. It should be avoided in patients with coronary artery disease because it can cause reflex tachycardia [2]. Hydralazine is useful in treatment of hypertension in pregnancy, along with methyldopa. A commonly reported adverse effect with prolonged treatment is a lupus-like reaction [4]. Headache, tachycardia, and palpitation are frequently reported ( $>10 \%$ patients), while flushing, hypotension, arthralgias, edema, nausea, and vomiting are less common (1-10\%) [5].

Hepatotoxicity with hydralazine therapy are not commonly reported. In a prospective study conducted between 1998 and 2007, among 1198 patients with acute liver, 133 cases were found to be due to liver injury caused by various drugs. Out of these, only 1 case was attributed to hydralazine use [5]. In another study, conducted over 12 years, out of 313 patients

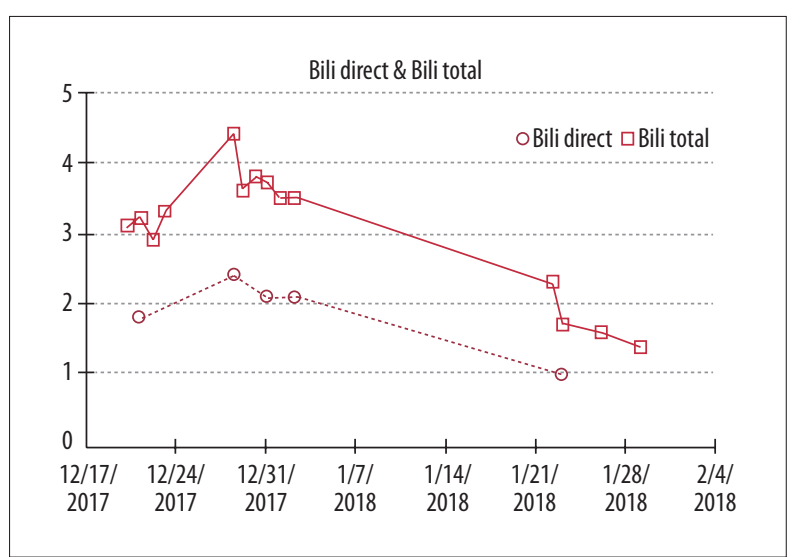

Figure 2. Trend of total serum bilirubin (solid line) and direct serum bilirubin (dotted line).

with liver injury due to drugs, no cases were attributed to hydralazine [6]. Two different types of injury pattern to the liver have been reported with hydralazine. There have been reports of hydralazine-induced hepatotoxicity with a short latency period ranging from 2 to 6 weeks [7], and there have also been case reports with latency periods ranging from 2 months to 1 year [8]. In our case, the patient was started on hydralazine 3 weeks before the onset of clinical symptoms and the onset of transaminitis. Patients with short latency have been described to have presented with fever, rash, and eosinophilia, and in these cases, rapid recovery has been described, while in cases with long latency, insidious onset was noted and biopsy of the liver demonstrated findings such as chronic hepatitis and fibrosis. Autoantibodies were also found to be associated with cases with long latency. The recovery is typically prolonged and these cases tend to be associated with lupuslike syndrome [8]. Autoantibodies to cytochrome P450 system (CYP1A2) were found in patients with hepatotoxicity due to dihydralazine (not available in the US), which is structurally similar to hydralazine [9]. Hydralazine hepatotoxicity has variable manifestation and can show a hepatocellular, cholestatic pattern of injury, hypersensitivity, or autoimmune features. An immunologic or a lupus-like reaction is believed to be the main mechanism of liver injury [10]. Our patient showed a cholestatic pattern of jaundice.

In most case reports, hepatic injury was reversed within few days to weeks of stopping hydralazine. Re-exposure to hydralazine has been reported to result in recurrence of hepatotoxicity [11]. In view of our patient's third admission within 2 months, he was not re-challenged with hydralazine, and since his liver function improved after discontinuation of hydralazine, autoantibodies to cytochrome P450 were not sent. Cardio-hepatic syndrome was ruled out since his left ventricular ejection fraction was normal. 


\section{Conclusions}

The hydralazine-induced cholestatic liver injury evident in our case showed clinical and biochemical recovery after discontinuation of the offending agent. Any new occurrence of hepatocellular injury after introduction of new medication should be carefully evaluated. Clinicians should be aware of

\section{References:}

1. Schroeder NA. The effect of 1-hydrasinophthalasine in hypertension. Circulation, 1952; 5: 28-37.

2. Harvey RA, Harvey PA, Mycek MJ: Lippincott's illustrated reviews: Pharmacology. $2^{\text {nd }}$ ed. Philadelphia: Lippincott Williams \& Wilkins, 2000; 190

3. Kandler MR, Mah GT, Tejani AM, Stabler SN: Hydralazine for essential hypertension. Cochrane Database Syst Rev, 2010; (8): CD004934

4. Iyer P, Dirweesh A, Zijoo R: Hydralazine induced lupus syndrome presenting with recurrent pericardial effusion and a negative antinuclear antibody. Case Rep Rheumatol, 2017; 2017: 5245904

5. Reuben A, Koch DG, Lee WM, Acute Liver Failure Study Group: Drug-induced acute liver failure: Results of a U.S. multicenter, prospective study. Hepatology, 2010; 52: 2065-76 hydralazine-induced hepatotoxicity so that such cases can be promptly identified to promote recovery.

\section{Conflict of interest}

None.

6. Devarbhavi H, Dierkhising R, Kremers WK et al: Single-center experience with drug-induced liver injury from India: Causes, outcome, prognosis, and predictors of mortality. Am J Gastroenterol, 2010; 105: 2396-404

7. Myers Jl, Augur NA: Hydralazine-induced cholangitis. Gastroenterology, 1984; 87: 1185-88

8. Hassan A, Hammad R, Cucco R, Niranjan S: Hydralazine-induced cholestatic hepatitis. Am J Ther, 2009; 16: 371-73

9. Bourdi M, Larrey D, Nataf J et al: Anti-liver endoplasmic reticulum autoantibodies are directed against human cytochrome P-450IA2. A specific marker of dihydralazine-induced hepatitis. J Clin Invest, 1990; 85: 1967-73

10. De Marzio DH, Navarro VJ: Hepatotoxicity of cardiovascular and antidiabetic medications. In: Kaplowitz N, DeLeve LD (eds.): Drug-induced liver disease. $3^{\text {rd }}$ ed. Amsterdam: Elsevier, 2013; 519-40

11. Forster HS: Hepatitis from hydralazine. N Engl J Med, 1980; 302: 1362 